\title{
3D Inkjet Printed Helical Antenna with Integrated Lens
}

\author{
Muhammad F. Farooqui, and Atif Shamim, Senior Member, IEEE
}

\begin{abstract}
The gain of an antenna can be enhanced through the integration of a lens, although this technique has traditionally been restricted to planar antennas due to fabrication limitations of standard manufacturing processes. Here, through a unique combination of $3 \mathrm{D}$ and $2 \mathrm{D}$ inkjet printing of dielectric and metallic inks respectively, we demonstrate a lens that has been monolithically integrated to a non-planar antenna (helix) for the first time. Antenna measurements show that the integration of a Fresnel lens enhances the gain of a 2-turn helix by around 4.6 dB, which provides a peak gain of about $12.9 \mathrm{dBi}$ at $8.8 \mathrm{GHz}$. The 3dB axial ratio (AR) bandwidth of the antenna with the lens is $5.5 \%$. This work also reports the complete characterization of this new process in terms of minimum features sizes and achievable conductivities. Due to monolithic integration of the lens through a fully printed process, this antenna configuration offers high gain performance by using a low cost and rapid fabrication technique.
\end{abstract}

Index Terms - 3D printing, inkjet printing, helical antenna, lens antenna

\section{INTRODUCTION}

$\mathrm{H}$ ELICAL antenna is one of the most widely used antennas due to its many attractive features such as high gain, wide bandwidth, circular polarization and the ability to radiate both omnidirectional and directive patterns [1]. The gain of the helical antenna can be increased by increasing the number of turns. However, the gain is not linearly proportional to the number of turns and after many turns, the addition of more turns has little effect [2]. Another method of increasing the gain of the helical antenna can be through integration of a dielectric lens.

Dielectric lenses are typically used to increase the directivity of the antennas because of their ability to focus electromagnetic waves in certain directions. Traditionally, lenses are used with planar or aperture antennas [3] due to the ease of integration and the limitations of manufacturing techniques. There are few reports of lens designs with nonplanar antennas [4,5], although the fabrication process has not been reported. Inkjet printing technology provides an attractive method for low cost and the rapid manufacturing of complex 3D objects that are very difficult, expensive and time consuming to fabricate using conventional techniques such as carving, molding and computer numerical control (CNC) machining [6]. Printing technology uses an additive process, which reduces material wastage, and by using a digitally

Manuscript received $\mathrm{x}, \mathrm{xxx}$.

M. F. Farooqui and A. Shamim are with the Electrical Engineering Department, King Abdullah University of Science and Technology, Thuwal 23955, KSA (e-mail: muhammad.farooqui@ kaust.edu.sa). controlled process, deposits a precise amount of material at a desired location. However, RF structures usually require integrated metal and dielectric, and current inkjet printing technology is still immature in depositing dielectric and metal at the same time. Inkjet printing of only dielectric materials has been used to fabricate RF structures [7,8]. On the other hand, inkjet printing of only metals has been shown to fabricate antennas on conventional planar substrates $[9,10]$. There are not many reports that show integration of inkjet printed metal and dielectric to realize RF structure. Other deposition methods such as spraying and painting have been used for the metallization of 3D printed dielectrics [11,12]. These methods are manual with less control over material deposition and layer thickness, and hence they cause significant material wastage. They are also not suitable for large volume manufacturing.

Here a unique combination of inkjet printing of dielectric and metal has been utilized for the realization of a novel 3D antenna configuration. For the first time, a helical antenna has been monolithically integrated to a dielectric lens through inkjet printing technology. As a result of the lens integration, a two-turn helical antenna provides a peak gain of $12.9 \mathrm{dBi}$ in the X-band. The characterization of the inkjet printed 3D dielectric material as well as inkjet printed metal on this dielectric in terms of minimum features sizes and conductivities has also been reported. This work shows the advantage of inkjet printing technology through which a dielectric lens, that has been typically limited to planar antenna designs, can easily be integrated to a helical structure.

\section{INKJET PRINTING CHARACTERIZATION}

In order to utilize the inkjet printing technology for the fabrication of RF structures, it is essential to know the constraints of this fabrication method in terms of dimensional resolutions and metal conductivities. The Stratasys ${ }^{\mathrm{TM}}$ Objet 260 Connex 1 3D printer has been used to inkjet print the dielectric material. The dielectric material used by the printer is a photo sensitive polymer named VeroBlackPlus ${ }^{\circledR}$. The material has a dielectric constant of 2.6 and a loss tangent of 0.023 . The printer has a layer resolution of $16 \mu \mathrm{m}$. In order to determine the lateral $x-y$ resolution of the 3D printer, narrow strips and gaps are printed, as shown in Fig. 1(a). This information is important for the realization of RF structures, as dimension become critical especially at higher frequencies. It is evident that the printer can reliably print feature with widths and gaps of around $300 \mu \mathrm{m}$. This resolution is sufficient for the fabrication of microwave components. 

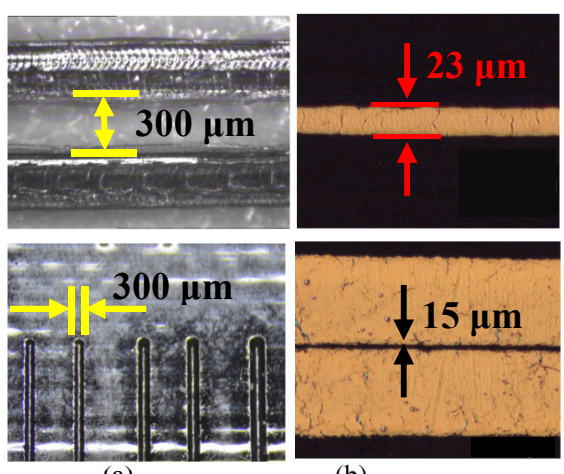

(b)

Fig. 1. (a) 3D printed gaps and strips. (b) Inkjet printed lines and gaps on 3D printed substrates.

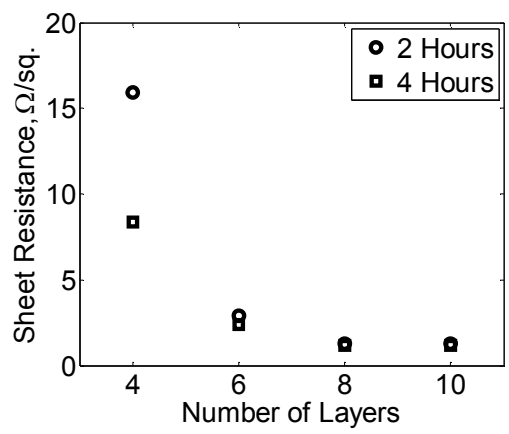

Fig.2. Sheet resistance of inkjet printed traces when sintered at $125^{\circ} \mathrm{C}$.

For the inkjet printing of metal, the Dimatix 2831 materials printer has been used. A commercial silver nanoparticle based ink from Sigma Aldrich (product 719048) with an average particle size of $150 \mathrm{~nm}$ is printed to form metal tracks on the 3D printed dielectric. As inkjet printing is highly dependent on the substrate type, it is necessary to characterize the ink on a particular substrate in order to obtain an accurate measure of the printing performance, as is done in [9] for the paper substrate. Before printing, it is necessary to treat the surface of the substrate with acetone in order to remove unwanted contaminants such as grease and oils. Metal lines and gaps are inkjet printed with $20 \mu \mathrm{m}$ drop spacing and $1 \mathrm{pl}$ drop volume cartridge as shown in Fig. 1(b). As made clear in the image, the minimum line width of around $23 \mu \mathrm{m}$ and gaps as narrow as $15 \mu \mathrm{m}$ are realizable using inkjet printing. The surface of the inkjet printed traces is slightly rough, as made visible in Fig. 1(b), which is due to the roughness of the $3 \mathrm{D}$ printed substrate. The measured root-mean-square roughness is 6.4 $\mu \mathrm{m}$. The conductivity of metal traces is critical for the performance of RF components. In order to get conductive traces, the printed traces are subjected to heat so that the nanoparticles fuse together to form continuous metal tracks. This process is termed sintering. The conductivity of the metal traces increases with the duration of the sintering, and temperature. Also more layers can be printed to achieve higher conductivities. Fig. 2 shows the sheet resistance of the printed metal traces against number of layers when traces are heated at $125{ }^{\circ} \mathrm{C}$ for 2 hours and 4 hours. As seen, the resistance decreases by printing more layers and also by increasing the sintering duration. A minimum sheet

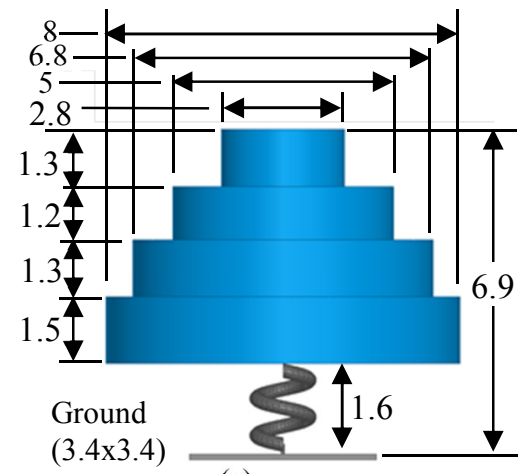

(a)

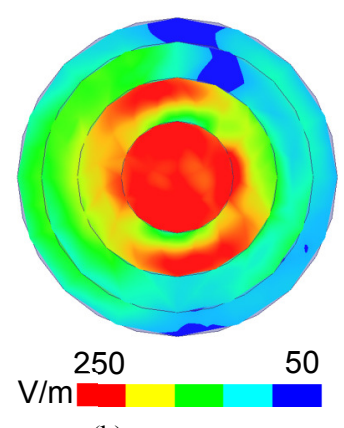

(b)
Fig. 3. (a) Helical antenna integrated to a Fresnel lens, dimensions in $\mathrm{cm}$. (b) Electrical field distribution over the surface of the lens

resistance of around $1.3 \Omega / \mathrm{sq}$. is achieved by printing 10 layers of silver ink giving a conductivity of around $3.8 \times 10^{5}$ $\mathrm{S} / \mathrm{m}$.

\section{ANTENNA Design AND SimUlations}

A 2 turn helical antenna has been designed with the radius and spacing between the turns meeting the criteria for end-fire circular polarized (CP) operation given in [1]. The diameter of the helix is $1 \mathrm{~cm}$, the wire diameter is $0.25 \mathrm{~cm}$ and the spacing between the turns is $0.7 \mathrm{~cm}$. The helix is then integrated into a dielectric Fresnel lens. The Fresnel lens structure consists of grooved zones such that the phase velocity of wave travelling within the zones is higher than in solid material. The depth of the grooves determines the phase difference between adjacent zones that should be equal to the desired phase compensation in order to transform the incoming spherical wave into a plane wave. The radius of each is zone is given by [13].

$$
R_{n}=\sqrt{\frac{2 n F \lambda_{0}}{P}+\left(\frac{n \lambda_{0}}{P}\right)^{2}}
$$

Where $\mathrm{n}$ is the zone number, $\lambda_{0}$ is the free space wavelength, $F$ is the focal length and $P$ is the number of different phases to implement phase compensation. The depth of the zones is given by

$$
d=\frac{\lambda_{0}}{4\left(\sqrt{\varepsilon_{r}}-1\right)}
$$

The radiation efficiency of the lens increases with $P$. Here, $P$ is equal to 4 , which means the phase needs to be corrected for every 90 degrees. For this quarter wave zone lens, successive groove depths of $d, 2 d$ and $3 d$ are used. A Fresnel lens has been designed with a focal length to diameter ratio $(F / D)$ of 0.04 , as shown in Fig. 3(a). The diameter of the lens has been chosen to be around twice of the wavelength in order to have reasonable dimensions of the lens when operating in the X-band.

The design has been simulated in Ansys HFSS. Fig. 3(b) shows the electric field pattern on the lens. The fields are 
concentrated towards the center of the lens, which shows that the lens is able to focus the incoming waves from the helical antenna. Fig. 4 (section IV) shows the $S_{11}$ of the antenna. As can be seen, the integration of the lens has little effect on the impedance bandwidth of the antenna, which is quite large, as is typically expected from a helical antenna. The simulated radiation pattern of the helical antenna before and after the lens integration at $9 \mathrm{GHz}$ is shown in Fig. 5 (section IV). The antenna radiates LHCP pattern. As can be seen, the gain of the antenna with the lens is around $14.4 \mathrm{dBi}$, which is enhanced by around $5 \mathrm{~dB}$ in comparison to the antenna without the lens. In order to achieve a similar gain, a 15 turn helical antenna having a length of around $11 \mathrm{~cm}$ will be required. The length of the helix with the Fresnel lens is just $6.9 \mathrm{~cm}$. However, the antenna becomes bulkier due to the integration of the lens. It should be noted that the cross-polarization levels have increased after the integration of lens. The possible reason for this increase is that any backward reflection from the lens due to air dielectric interface would be partially reflected back by the ground in the forward direction with the opposite sense of polarization, and thereby would add to the cross polarization levels.

\section{FABRICATION AND RESULTS}

The antenna has been manufactured by a combination of 3D inkjet printing of dielectric and 2D inkjet printing of metal. The helical structure along with the lens has been monolithically manufactured using a $3 \mathrm{D}$ inkjet printer. This shows a major advantage of 3D printing, as complex structures can be fabricated rapidly with the precise layer-bylayer deposition of material through a digitally controlled process. The print time for this 3D geometry is 5 hours and 21 minutes with the available printer. An industrial grade printer will take $40 \%$ less time to print the same geometry [14]. After 3D printing of helix and lens, the helix is metallized using inkjet printing of silver nanoparticle ink. Metal ink is deposited in $1 \mathrm{pl}$ drop volumes at the desired locations on the helix through a computer controlled process, and thus minimizes material wastage and increasing speed. Since the helix is a $3 \mathrm{D}$ structure and metal inkjet printing is a $2 \mathrm{D}$ process, the metallization process involves three cycles of inkjet printing. After each cycle, the helix is rotated by about $120^{\circ}$. Also, only the outer half portion of the helical structure is metalized as can be seen in Fig. 6, due to printing limitations. The same metallization geometry has been used in the simulation and it does not have significant effect on the antenna performance, in comparison to the helix made of metal wire. This approach of only printing the outside of the helix further saves material and cost without affecting the antenna's performance. The inkjet metallization took 21 minutes. For mechanical support, two vertical posts have also been attached to the lens after the metallization of the helix as shown in Fig. 6, which rest on the ground plane. The ground has been inkjet printed separately because of the inkjet printer's limited work area, which cannot accommodate the

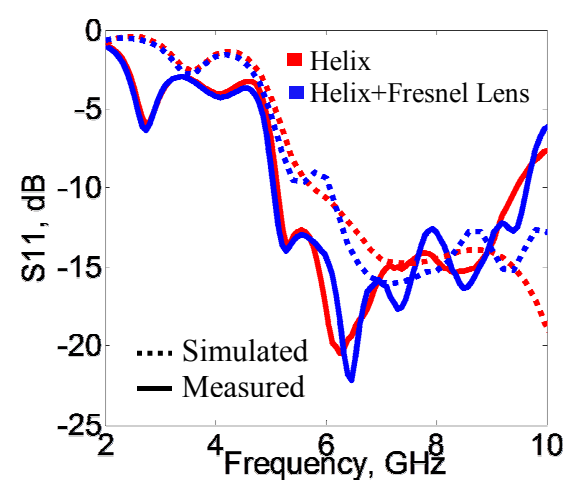

Fig. 4. Simulated and measured S11

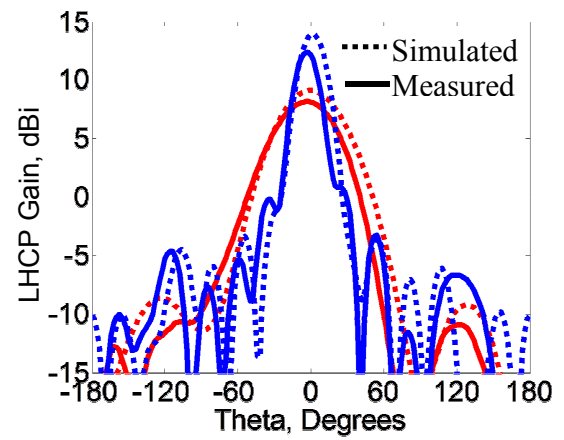

(a)

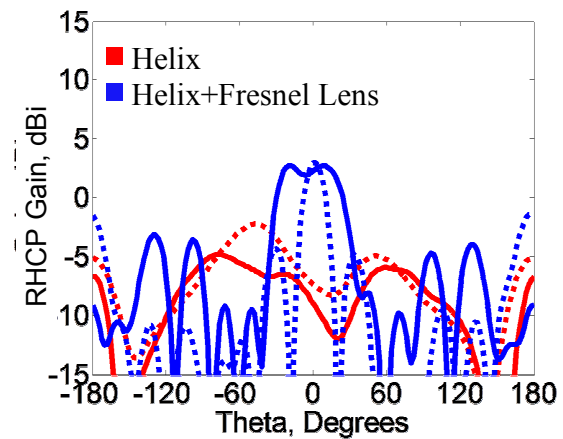

(b)

Fig. 5. Measured gain patterns with and without lens at $9 \mathrm{GHz}$ and $\mathrm{Phi}=0^{\circ}$. (a) LHCP. (b) RHCP.

complete structure. However, this will not be an issue if larger printers with more work volumes are used. After attaching the ground plane, the SMA connector is mounted on the antenna for measurements. The $\mathrm{S}_{11}$ of the antennas are measured using Agilent's E8361C PNA series network analyzer. The results are shown in Fig. 4. The measured results conform to the results of the simulation. Slight discrepancy can be due to connector mounting errors and the effect of metal surface roughness. The gain patterns of the antenna are measured in Satimo StarLab near field measurements system. The measured gain patterns at $9 \mathrm{GHz}$ are shown in Fig. 5. The measured peak gain of the helical antenna with Fresnel lens is $12.9 \mathrm{dBi}$ at $8.8 \mathrm{GHz}$ which give an increase in gain of around $4.6 \mathrm{~dB}$ as compared to the helix without the lens. There is a 1 $\mathrm{dB}$ decrease in gain in comparison to the simulation. This decrease can be due to the effect of connector losses and the surface roughness of the inkjet-printed metal. The measured $3 \mathrm{D}$ radiation patterns at $9 \mathrm{GHz}$ are shown in Fig. 7 and demonstrate an increase in the directivity after lens integration. The measured axial ratios of the helical antenna 

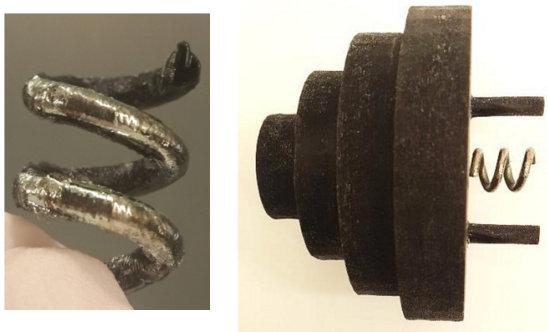

Fig. 6. Fabricated antennas without the ground planes.

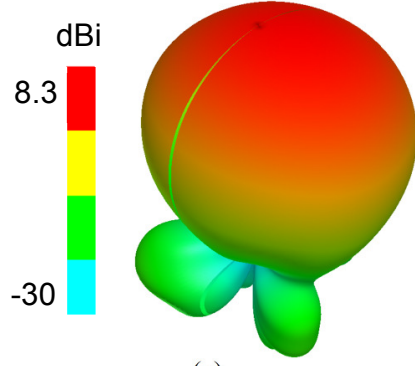

(a)

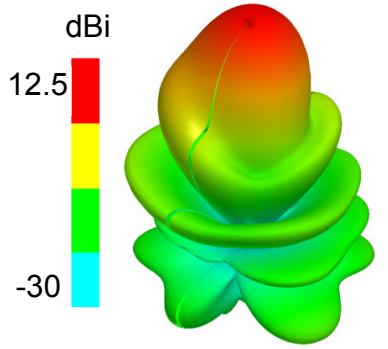

(b)
Fig. 7. Measured 3D radiation patterns at $9 \mathrm{GHz}$. (a) Helical antenna. (b) Helical integrated to Fresnel lens

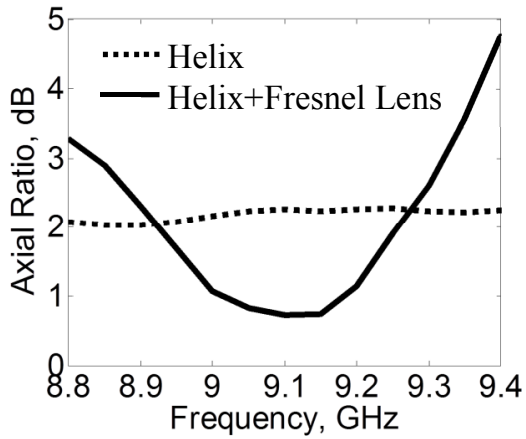

Fig. 8. Measured axial ratio.

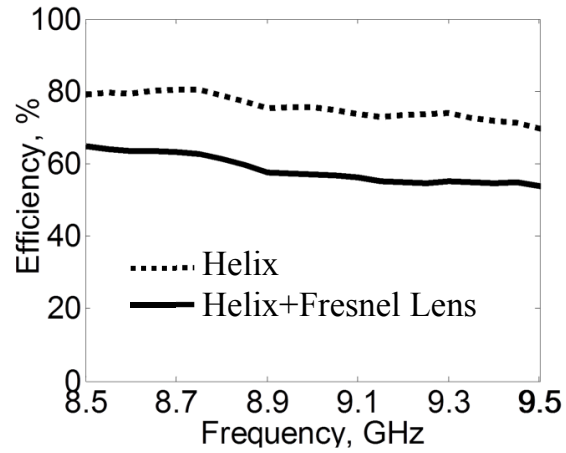

Fig. 9. Measured efficiency.

with and without the lens are shown in Fig. 8. The $3 \mathrm{~dB}$ axial ratio (AR) bandwidth is around $5.5 \%$. The AR bandwidth has decreased after the integration of the lens due to the frequency dependent properties of the lens antenna. The measured efficiency of the antennas is shown in Fig. 9. Inkjet printed antennas have typical efficiencies of around 50-70\% $[15,16]$.
The efficiency of our design is $65 \%$ which is on the higher side as compared to inkjet printed antennas and comparable to traditional non-3D printed lens antennas whose efficiency can vary from about $50 \%-80 \%[17,18]$.

\section{CONCLUSION}

In this work, a 2 turn helical antenna monolithically integrated with a lens through 3D inkjet printing is presented. The integration of the lens has resulted in the gain enhancement of of $4.6 \mathrm{~dB}$, yielding a peak gain of $12.9 \mathrm{dBi}$. The proposed antenna configuration has the potential to provide a high gain $\mathrm{CP}$ radiation characteristics. This work shows the utility of additive printing technologies to realize complex 3D RF components that are very difficult and expensive to realize using conventional manufacturing techniques.

\section{REFERENCES}

[1] J. D. Kraus, "Helical beam antennas for wide-band applications" IRE Proc., vol. 36, issue 10, 1948

[2] H. E. King and J. L. Wong, "Characteristics of 1 to 8 wavelength unifom helical antennas," IEEE Trans. Antennas Propag., vol. ap-28, no. 2, 1980

[3] J. Thornton and K.-C. Huang, Modern Lens Antennas for Communications Engineering, 1st Ed., John Wiley \& Sons, Inc., 2013

[4] J. Yamauchi and H. Nakano, "Theoretical and experimental investigations on focusing properties of the Fresnel Zone lens and plate," IEEE Int. Symp. Antennas Propag., 1999.

[5] M.F. Farooqui, A. Shamim, "A 3D printed helical antenna with integrated lens," IEEE Int. Symp. Antennas Propag., 2015

[6] I. Gibson, D. W. Rosen, B. Stucker, Additive Manufacturing Technologies: Rapid Prototyping to Direct Digital Manufacturing, Springer, 2010

[7] M. Liang, W.-R. Ng, K. Chang, K. Gbele, M. E. Gehm, and H. Xin, "A 3-D luneburg lens antenna fabricated by polymer jetting rapid prototyping," IEEE Trans. Antenna and Propag., vol. 62, no. 4, 2014

[8] J. H. Barton, C. R. Garcia, E. A. Berry, R. Salas, and R. C. Rumpf, "3-D printed all-dielectric frequency selective surface with large bandwidth and field of view," IEEE Trans. Antennas Propag., vol. 63, no. 3, 2015.

[9] B. S. Cook, and A. Shamim, "Inkjet printing of novel wideband and high gain antennas on low-cost paper substrate," IEEE Trans. Antennas Propag, vol .60, no. 9, 2012, 4148-4156

[10] H R. Raad, A. I. Abbosh, H. M. Al-Rizzo, and D. G. Rucker, "Flexible and compact AMC based antenna for telemedicine applications," IEEE Trans. Antennas Propag., Vol. 61, No. 2, February 2013

[11] O. S. Kim, "3D printing electrically small spherical antennas," IEEE Int. Symp. Antennas Propag., 2013.

[12] J. M. Floch, B. El Jaafari, and A. El Sayed Ahmed, "New compact broadband GSM/UMTS/LTE antenna realised by 3D printing," 9th European Conf. Antennas Propag., (EuCAP), 2015 pp. 1-4.

[13] H. D. Hristov, Fresnel zones in wireless links, zone plate lenses and antennas, Artech House, Boston, 2000

[14] [Online]. Available. http://www.stratasys.com/3d-printers/productionseries/objet1000-plus

[15] H. F. Abutarboush, M. F. Farooqui, A. Shamim, "Inkjet-printed wideband antenna on resin-coated paper substrate for curved wireless devices," IEEE Antennas Wireless Propag. Lett., 2015

[16] H. Saghlatoon, T. Björninen, L. Sydänheimo, M. M. Tentzeris, and L. Ukkonen, "Inkjet- printed wideband planar monopole antenna on cardboard for RF energy-harvesting applications," IEEE Antennas Wireless Propag. Lett., vol. 14, 2015.

[17] M. K. T. Al-Nuaimi, W. Hong, and Y. Zhang, "Design of highdirectivity compact-size conical horn lens antenna" IEEE Antennas Wireless Propag. Lett., vol. 13, 2014

[18] O. Yurduseven, D. Cavallo and A. Neto, "Wideband dielectric lens antenna with stable radiation patterns fed by coherent array of connected leaky slots," IEEE Trans. Antennas Propag., vol. 62, no. 4, 2014 